Acta Crystallographica Section A

Foundations of

Crystallography

ISSN 0108-7673

Received 30 July 2004

Accepted 13 October 2004

(C) 2005 International Union of Crystallography Printed in Great Britain - all rights reserved

\section{X-ray studies of the phases and phase transitions of liquid crystals}

\author{
Paul S. Clegg
}

School of Physics, University of Edinburgh, Mayfield Road, Edinburgh EH9 3JZ, Scotland. Correspondence e-mail: pclegg@ph.ed.ac.uk

\begin{abstract}
A short review is given of recent X-ray diffraction studies of the phases and phase transitions of thermotropic liquid crystals. The areas covered are twistgrain-boundary phases, antiferroelectric phases studied with resonant X-ray diffraction and smectic phases within gel structures. In all areas, X-ray diffraction has played a key role. Nonetheless, open questions remain: the nature of the smectic $\mathrm{C}$ variant of the twist-grain-boundary phase, the origin of antiferroelectric phases, and whether novel Bragg glass states exist for smectic $\mathrm{A}$ gel samples.
\end{abstract}

\section{Introduction}

It was first suggested that smectic liquid crystals are ordered in layers, based on observations of large-scale defect structures in the 1920 's as described by de Gennes \& Prost (1993). However, the novel structure of the smectic A (SmA) phase (Als-Nielsen et al., 1980) and the character of the nematic (N) to SmA transition (Davidov et al., 1979) were established using $\mathrm{X}$-ray diffraction complemented by other techniques. X-ray diffraction also played a decisive role in elucidating the properties of the SmA-smectic C (SmC) transition (Safinya et al., 1980), where the molecules acquire a tilt with respect to the layer normal. Similarly, it was an important technique for the SmA-chiral SmC (SmC $)$ transition (Shashidhar et al., 1988). Major reviews of X-ray studies of liquid crystals have been carried out by Pershan (1988) and Seddon (1998). The latter gives a comprehensive list of reviews of structural studies of liquid crystals. A recent review by Kumar (2001) focuses on experimental techniques. The selection of topics included here is intended to be representative rather than complete. The topics concern the perturbation of SmA and SmC phases by twist, dipole moments and local deformations. Novel phases and phase-transition behaviour result.

$\S 2$ focuses on twist-grain-boundary (TGB) phases especially those with SmC order. The TGB phase was proposed by Renn \& Lubensky (1988) and discovered by Goodby et al. (1989a,b). These are smectic phases where arrays of defects form part of the ordered structure. The layer order can have SmA, SmC or $\mathrm{SmC}^{*}$ structure. The defects are a means of incorporating the twist of chiral molecules into a layered phase. More recently, large-scale superstructures have been observed in the plane perpendicular to the helical axis (Pramod et al., 1997; Ribeiro et al., 1999). The nature of the SmC and SmC* TGB structures and their relationship to larger scale superstructures are still open issues (Brunet et al., 2002).

$\$ 3$ concerns the family of antiferroelectric phases. Antiferroelectricity in stacks of two-dimensional liquids was not predicted before its discovery. Initial experimental signatures were not immediately associated with this phenomenon (Hiji et al., 1988; Furukawa et al., 1988). Subsequently, a series of antiferroelectric phases were discovered (Fukuda et al., 1994). These include both commensurate and incommensurate stacking sequences. Resonant X-ray scattering has enabled these sequences to be determined (Mach et al., 1998). The structures occur for materials with large tilts, large dipole moments and no cholesteric phase. The fundamental question of the origin of the phases is still not resolved (Lagerwall et al., 2003).

$\$ 4$ covers studies of the phase transitions of liquid crystals confined in gels. Early experiments were carried out for the N-SmA transition in rigid aerogels (Clark et al., 1993). Enhanced anomalous elastic effects and a smectic Bragg glass state were later predicted (Radzihovsky \& Toner, 1999). Studies in rigid aerogels (Bellini et al., 2001) and soft aerosil gels (Park et al., 2002) have been made to test this model. Smectic Bragg glass signatures from aerogel samples (Bellini et al., 2001) have not reappeared with aerosil samples under conditions where they should be more evident (Leheny et al., 2003; Clegg, Stock et al., 2003).

\section{Twist-grain-boundary phases}

De Gennes (1972) identified an analogy between the Meisner state of a superconductor and the smectic state of a liquid crystal. In the Meisner state, a magnetic field is expelled from the solid; equivalently, twist is expelled from the smectic state. Abrikosov (1957) predicted a vortex solid state in type II superconductors in an applied magnetic field. De Gennes (1972) hinted at and Renn \& Lubensky (1988) described the analogous phase for a liquid crystal. In the Abrikosov vortex lattice, a magnetic field penetrates the sample at defects (vortices) which themselves order in a regular array. On heating, the state returns to a normal metal via a vortex liquid 
phase. Twist is introduced into a smectic when the molecules have chirality and a single enantiomer is present. This contributes a term

$$
f_{\mathrm{Ch}}=-h \hat{\mathbf{n}} \cdot \nabla \times \hat{\mathbf{n}}
$$

to the Frank free-energy density (which describes the energy cost of distortions to the orientational order). Here, $h$ is the strength of the twist and $\hat{\mathbf{n}}$ is the nematic director. Renn \& Lubensky (1988) detailed a twist-grain-boundary state with blocks of well ordered smectic separated from one another by walls of regularly spaced dislocations. The layer normal is offset by an angle $\Delta$ from one block to the next with an axis parallel to the planes. The angle is given by $d=2 \ell_{d} \sin (\Delta / 2)$, where $d$ is the thickness of the smectic layers and $\ell_{d}$ is the separation between screw dislocation lines. The structure thus has a helical axis and twist has been incorporated into the phase. Chiral liquid crystals often have a cholesteric phase at high temperatures; a full pursuit of the analogy would suggest that a twist line liquid phase, $\mathrm{N}_{\mathrm{L}}^{*}$, should occur between the cholesteric phase and the twist-grain-boundary phase in parallel to the vortex liquid phase. Kamien \& Lubensky (1993) have discussed the structure of the $\mathrm{N}_{\mathrm{L}}^{*}$ phase, concluding that the screw dislocation lines should themselves have cholesteric order.

\subsection{Twist-grain-boundary $\mathrm{SmA}$ and $\mathrm{N}_{\mathrm{L}} N_{r m L}^{*}$ phases}

The TGB phase was discovered by Goodby et al. (1989a,b) following the theoretical impetus. For the X-ray studies on oriented samples, which followed, very long correlation lengths were required to see many ordered blocks of twistgrain-boundary phase. Sample holders tens of micrometres thick with rubbed surfaces were used to create the orientational order. Detailed studies of the TGB SmA (TGBA) phase and the TGBA to $\mathrm{N}_{\mathrm{L}}^{*}$ transition were carried out by Navailles et al. (1998). The TGBA phase was found to be made up of a series of blocks of SmA order each with a layer normal which is perpendicular to the helical axis (as implied by Fig. 1). The layer normal rotates in discrete steps from one block to the next at a grain boundary. It was shown that the size of SmA blocks, $\ell_{b}$, in the TGBA phase was approximately equal to the distance between the screw dislocations, $\ell_{d}$, in the grain boundaries as predicted (Renn \& Lubensky, 1988).

Measurements using ac-calorimetry revealed a large $\Delta C_{p}$ peak (Chan \& Garland, 1995) consistent with the cholesteric phase evolving into a twisted line liquid phase $\mathrm{N}_{\mathrm{L}}^{*}$. The warming of the TGBA phase into the $\mathrm{N}_{\mathrm{L}}^{*}$ phase is the analogue of the Abrikosov vortex lattice melting and here was shown to be a first-order transition. The transition from the vortex solid phase to the vortex liquid phase TGBA to $\mathrm{N}_{\mathrm{L}}^{*}$ was found to be marked by the helical twist angle becoming incommensurate (Navailles et al., 1998) and a steady decrease in the SmA order parameter (here the parallel correlation length, $\xi_{\|}$, is reported to remain unchanged). Further studies of the transition from the TGBA to $\mathrm{N}_{\mathrm{L}}^{*}$ phase have been made by Ybert et al. (2003). They found the temperature dependence of the smectic correlation length consistent with melting via the $\mathrm{N}_{\mathrm{L}}^{*}$ phase.
The different result to that reported above is probably due to an increase in the wavevector resolution for the second study.

\subsection{Twist-grain-boundary $\mathrm{SmC}$ and $\mathrm{SmC}^{*}$ phases}

Renn \& Lubensky (1991) predicted a SmC variant of the twist-grain-boundary phase where the molecules acquire a tilt angle in the plane perpendicular to the helical axis. Further complications can occur. The SmC phase is modified by chiral molecules to give the $\mathrm{SmC}^{*}$ phase. The azimuthal angle associated with the tilt precesses from one plane to the next. This creates a helix with a pitch $\sim 1 \mu \mathrm{m}$. With chiral molecules, there is the possibility of a TGB phase with twist along two orthogonal axes as predicted by Renn (1992) for the TGB $\mathrm{SmC}^{*}\left(\mathrm{TGBC}^{*}\right)$ phase. Here the SmC slabs are grouped in larger clusters called helislabs with a shared helical axis for $\mathrm{SmC}^{*}$ order.

The TGB SmC (TGBC) phase was discovered by Nguyen $e t$ al. (1992). Historically, X-ray measurements on oriented samples were made on the TGBC phase before the TGBA phase. On cooling the sample from the $\mathrm{N}^{*}$ phase into the TGBC phase, the continuous ring of scattering from shortrange smectic correlations is seen to become strongly modulated in intensity (Navailles et al., 1993). The period of the modulation varies discontinuously on cooling. Each peak on the ring is due to scattering from one set of blocks of smectic order. The orientation of these blocks jumps at the grain boundaries by angles commensurate with the pitch of the helix. Both the pitch of the helix and the size of the blocks may vary with temperature.

It was predicted by Renn \& Lubensky (1991) that the layer normal would be perpendicular to the axis of the helix. Experiments showed that this is not the case (Navailles et al., 1995), at least not for this variant of the TGBC phase. The

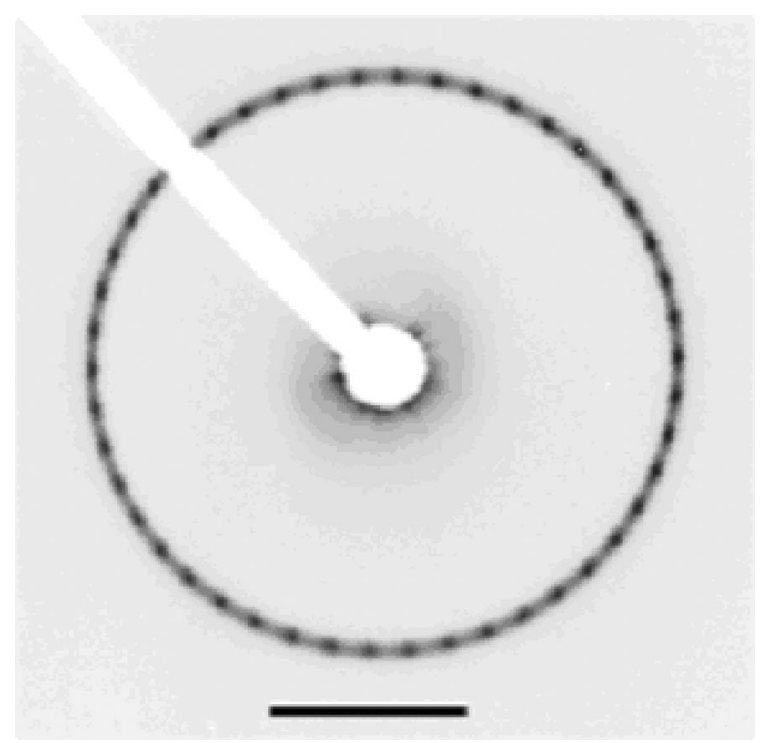

Figure 1

The modulated X-ray intensity due to the TGBA phase. In this case, the ring has 64 spots. Reprinted figure with permission from Navailles $e$ al. (1998). Phys. Rev. Lett. 81, 4168-4171. Copyright (1998) by the American Physical Society. 
TGBC phase studied in Bordeaux and described above is designated B-TGBC. The situation is shown in Fig. 2 with the layer normal at angle $\omega_{L}$ to the direction of the molecules (which are orthogonal to the helical axis). The tilt angle, $\omega_{L}$, grows from zero with a power law on entering the TGBC phase. This observation implies that the grain boundaries are more complicated than a grid of screw dislocations. Based on these results, a model was created in which it was demonstrated that, in the TGBC structure described by Renn (1992), the director orientation can barely vary across a SmC block (Dozov, 1995). This limits the amount of twist that can be accommodated in this defect structure. Dozov (1995) proposed a structure with a component of the layer normal in the TGB axis direction and with melted grain boundaries. With this structure, the director can then precess across a block while maintaining a constant angle to the planes.

A further anomaly with the B-TGBC structure is that it has only been observed as a commensurate structure (the ratio of the helical pitch $\Lambda=2 \pi \ell_{b} / \Delta$ to the block thickness $\ell_{b}$ is always an integer). With an incommensurate TGBC phase, the plane spacing would be observed to decrease without the discrete spots developing. The fact that the structure is always commensurate implies that there are surprisingly long-range interactions between the $\mathrm{SmC}$ blocks or between the defects in the boundaries (Galerne, 2000).

Subsequently, a range of other TGBC and TGBC* phases were discovered. The most prominent feature with the new phases is that order is observed on $\mu \mathrm{m}$ length scales in the plane perpendicular to the TGB axis. The first to be found was

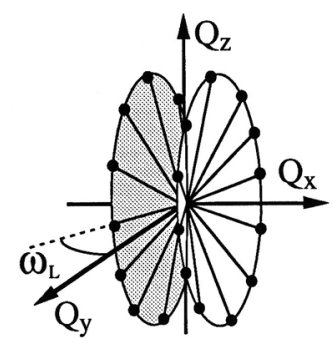

(a)

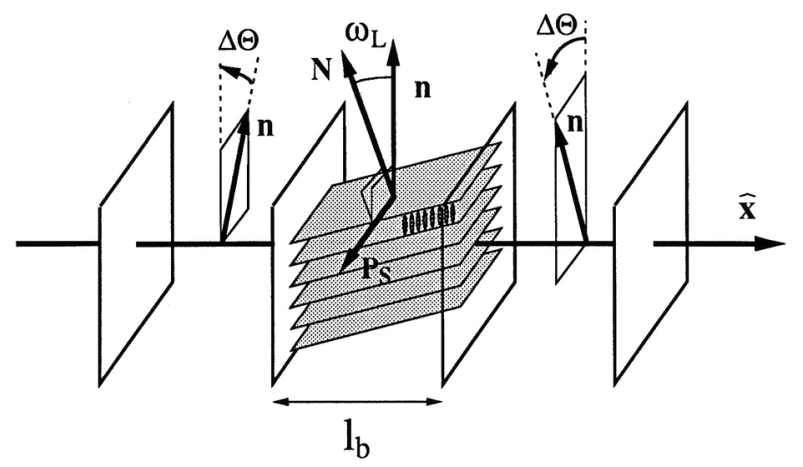

(b)

Figure 2

The structure of the TGBC phase as seen in $(a)$ reciprocal space and $(b)$ direct space. The smectic layers are tilted by angle $\omega_{L}$; the director field and layer normal for the $i$ th slab are $n_{i}$ and $N_{i}$, respectively. Adapted with permission from Petit et al. (1996). Europhys. Lett. 36, 185-190. reported by Pramod et al. (1997). The TGBC* phase was observed in a binary mixture and exhibited a square grid pattern of in-plane undulations; it is designated U-TGBC*. Subsequently, X-ray studies were made on a single component TGBC material which also exhibited a square superstructure in plane (Ribeiro et al., 1999). The spots from the SmC blocks were substantially broadened in the TGB axis direction suggesting that the direction of the plane normal was varying within a block. It has not been determined whether the structure is commensurate. This phase was characterized by a team in Strasbourg and hence is denoted the S-TGBC phase. A brief account has also been published (Brunet et al., 2002) of a TGBC phase in which the normal to the plane is perpendicular to the TGB axis as predicted by Renn \& Lubensky (1991). In this case, six spots are observed from the SmC blocks and there is a simultaneous hexagonal grid pattern.

Galerne (2000) and subsequently Brunet et al. (2002) have attempted to unify these results by extending Renn's model of the TGBC* phase (Renn, 1992). Galerne has shown that the helislab structure remains possible when the director is predominantly perpendicular to the TGB axis. For SmC* blocks, the component of the director in the smectic plane, $\mathbf{c}$, precesses around the layer normal, $\mathbf{N}$. This can occur if there is a modulation of $\mathbf{n}$ with wavevector $\mathbf{q}$ in the $y z$ plane and a simultaneous modulation of $\mathbf{N}$ with the same wavevector but in the $x z$ plane. The second modulation must have a phase shift of $\pi / 2$ from the first. This construction gives $\mathrm{SmC}^{*}$ order but requires that there is a compression and dilation of the layers. This could be possible for small smectic blocks and indeed is consistent with the peak broadening observed for the $\mathrm{S}$-TGBC phase. It is shown that angular lock-in may occur due to elastic interactions between disclination lines. If so, the helislabs will be commensurate and the disclination lines from different boundaries will overlay each other. Disclination lines scatter light and so will be visible using microscopy. For large commensurate angles, superstructures will be observed. This is consistent with some of the known behaviour of TGBC* materials.

Brunet et al. (2002) introduced a perspective on TGBC and $\mathrm{TGBC}^{*}$ structures based on experience with $\mathrm{SmC}^{*}$ materials sandwiched between glass plates. The model presented is somewhat similar to those of Renn (1992) and Galerne (2000) except that the helislabs are the same thickness as the smectic blocks. The blocks are polarization-splayed smectic slabs with boundaries that have a lattice of unwinding lines on either side. Overlapping unwinding lines give the observed superstructures. The experimental results might be consistent with the models of Galerne (2000) and Brunet et al. (2002) with different size helislabs for the different structures observed.

\section{Resonant X-ray studies of antiferroelectric phases}

Chiral molecules reduce the symmetry of the SmC phase by facing, on average, a particular in-plane direction (Meyer et al., 1975). For molecules with a transverse dipole moment (which is the usual case), this gives the ferroelectric $\mathrm{SmC}^{*}$ phase. The structure has a slight precession of the tilt giving a helix with a 
pitch $\sim 1 \mu \mathrm{m}$. An antiferroelectric $\mathrm{SmC}_{\mathrm{A}}^{*}$ phase was discovered in the late 1980's during attempts to synthesize new ferroelectric materials (Hiji et al., 1988; Furukawa et al., 1988) as described by Fukuda et al. (1994). The tilt and transverse dipole moment directions alternate close to $\pi$ from one plane to the next, the deviation being due to the long helical pitch (Fig. 3). Further cooling from this phase leads directly to either a solid crystal phase or a hexatic phase. Subsequently, new phases have been discovered which are stable both at higher temperature than the $\mathrm{SmC}^{*}$ phase $\left(\mathrm{SmC}_{\alpha}^{*}\right)$ and also in the temperature range between the $\mathrm{SmC}^{*}$ and $\mathrm{SmC}_{\mathrm{A}}^{*}$ phases (Fukuda et al., 1994). In the latter range, two phases have been identified. One of the most powerful techniques for unravelling the nature of these phases is resonant X-ray diffraction (Mach et al., 1998). It has been possible to discriminate between plane ordering configurations for stacked planes of liquids. X-ray reflectivity studies have also been employed to study the stacking sequence of thin films (Fera et al., 2001). Conventional X-ray diffraction studies of higher-order reflections have probed the interdigitation of smectic layers (Takanishi et al., 1995). The observed phases are now the subject of theoretical research.

\subsection{Resonant X-ray diffraction}

$\mathrm{X}$-ray scattering at energies close to an atomic absorption edge reveals information about the environment of that atom. It is called resonant scattering and it has been used for two decades to study solid crystals. A key feature was demonstrated by Templeton \& Templeton (1982), who showed that resonant scattering depends on the polarization direction for anisotropic molecules. The reason for this is that resonant scattering involves virtual transitions of the inner-shell electron to an intermediate state (Als-Nielsen \& McMorrow, 2001). The resonant effect probes the unoccupied states that are connected to the inner shell by an electric dipole transition. Owing to selection rules, the possibility of transitions

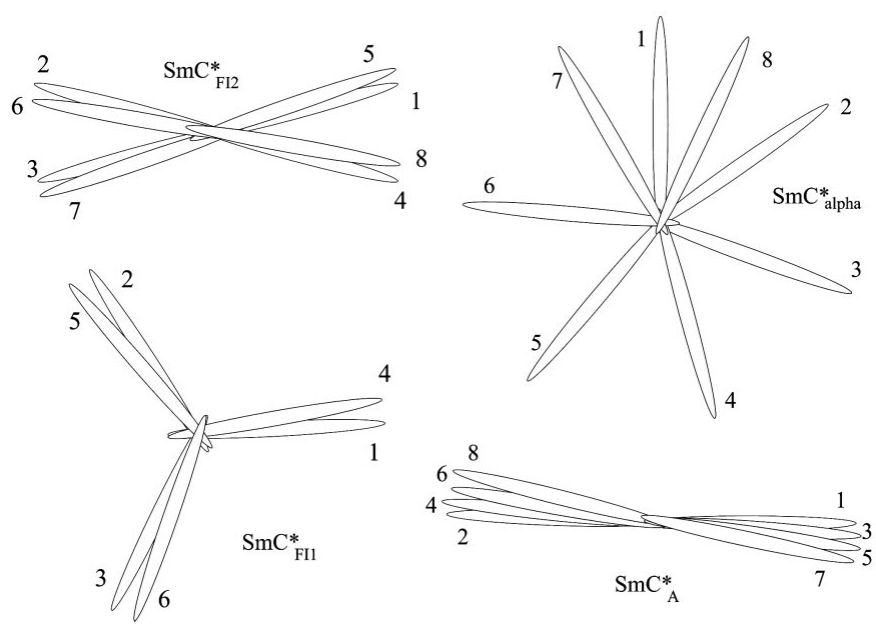

Figure 3

Variation of the azimuthal angle of molecules for the antiferroelectric subphases. The numbers indicate the plane sequence. Adapted from Hirst et al. (2002). depends on the relative orientation of the X-ray electric field and the orbital. Additional reflections can occur if the anisotropy of the electron environment exhibits a new periodicity.

Dmitrienko (1983) considered how resonant scattering would modify the signature of glide planes and screw axes. The form factor and polarization conditions for the new scattering at previously forbidden reflections were calculated. Levelut \& Pansu (1999) extended the studies of Dmitrienko: they calculated the tensor structure factor for different incommensurate helical phases and for specific plane sequence commensurate structures. In practice, $\sigma$-polarized radiation is usually used. The first-order reflections are then predicted to be $\pi$ polarized whereas the second-order reflections are elliptically polarized. In this case, the balance between $\sigma$ and $\pi$ depends on $\sin (\theta)$, where $2 \theta$ is the scattering angle, leading to predominantly $\sigma$ polarization. Levelut \& Pansu (1999) note that models can be discriminated using polarization analysis and that distortion angles can be found from the relative intensities of the resonant reflections.

A synchrotron X-ray source is essential to obtain the continuous spectrum of polarized radiation required for resonant diffraction. The low energies used for the sulfur $K$ edge are strongly scattered by air. This implies that the whole apparatus needs to be evacuated.

\subsection{Antiferroelectric phases}

Fig. 3 shows the range of different antiferroelectric phases that can occur for chiral materials with a SmC phase. The most elaborate phase sequence for a chiral molecule studied using resonant X-ray diffraction is for 10OTBBB1M7, which has the sequence I-SmA-SmC $C_{\alpha}^{*}-\mathrm{SmC}^{*}-\mathrm{SmC}_{\mathrm{F} 2}^{*}-\mathrm{SmC} \mathrm{F}_{\mathrm{F} 1}^{*}-\mathrm{SmC}_{\mathrm{A}}^{*}-\mathrm{K}$ on reducing the temperature (Mach et al., 1998, 1999; Hirst et al., 2002). For materials with fewer phases, the order of those that remain is retained. The $\mathrm{SmC}_{\mathrm{F} 1}^{*}$ and $\mathrm{SmC}_{\mathrm{FI} 2}^{*}$ are three and four plane antiferroelectric phases. The resonant diffraction results have been compared with existing theoretical models. Ising models had previously been used to explain the antiferroelectric plane sequences (Fukuda et al., 1994). These required interlayer angles to be 0 or $\pi$. By contrast, a clock model allows for any constant angle increment between planes (Čepič \& Žekš, 1995; Lorman, 1995; Pikin et al., 1995; Roy \& Madushudana, 1996). Based on the experimental results, more detailed models are being developed.

As described above, for X-rays close in energy to an absorption edge, the scattering depends on the photon polarization relative to the orientation of the molecule containing the electron. Determination of the in-plane orientation of molecules in antiferroelectric phases becomes possible. Mach et al. (1998) presented the first attempt in this direction and they showed clearly that different antiferroelectric structures had different stacking sequences in terms of azimuthal orientation (see Fig. 3). These studies involved chiral molecules incorporating sulfur, which have an accessible $K$ edge. Subsequent studies involved selenium (Matkin et al., 2001). In addition to the antiferroelectric stacking sequences, 
these materials have a helical pitch in the $\sim 1 \mu \mathrm{m}$ range. This research was a significant advance for the field. The structure of $\mathrm{SmC}_{\alpha}^{*}$ was here determined for the first time (Mach et al., 1998). These measurements were able to rule out Ising-type models for the antiferroelectric phases in favour of a clock model.

Mach et al. (1999) have studied the $\mathrm{SmC}_{\alpha}^{*}, \mathrm{SmC}_{\mathrm{F} 2}^{*}, \mathrm{SmC}_{\mathrm{F} 11}^{*}$, $\mathrm{SmC}_{\mathrm{A}}^{*}$ phases in liquid crystals containing sulfur while using polarization analysis to test the predictions of the clock model. The resonant peaks were often observed to be split (see Fig. 4) due to the coupling between the short plane sequence and the long helical pitch. In the clock model, the first-order satellites are predicted to be $\pi$ polarized. By contrast, the second-order reflections are $\sigma$ polarized. This was observed, confirming the polarization predictions for the first time.

In spite of many years of research, the $\mathrm{SmC}_{\alpha}^{*}, \mathrm{SmC}_{\mathrm{F} 2}^{*}$, $\mathrm{SmC}_{\mathrm{FI} 1}^{*}, \mathrm{SmC}_{\mathrm{A}}^{*}$ phases are still not fully understood. Resonant $\mathrm{X}$-ray scattering showed the stacking sequences for the first time. Following studies of MHPOBC, it was implied (Lagerwall et al., 2003) that the intervention of the $\mathrm{SmC}^{*}$ phase between $\mathrm{SmC}_{\alpha}^{*}$ and $\mathrm{SmC}_{\mathrm{F} 2}^{*}$ phases could be due to sample degradation or impurity effects. The $\mathrm{SmC}_{\alpha}^{*}$ phase follows in temperature below the SmA phase. In this phase, the tilt angle is changing rapidly from zero as the temperature is lowered. The plane sequence is incommensurate with the long helical pitch. Hirst et al. (2002) provided new information concerning the $\mathrm{SmC}_{\alpha}^{*}-\mathrm{SmC}^{*}-\mathrm{SmC}_{\mathrm{FI} 2}^{*}$ phase sequence with a $\mathrm{SmC}^{*}$ phase that was stable for a long temperature range. The periodicity varied from about 10 layers at higher temperature $\left(\mathrm{SmC}_{\alpha}^{*}\right)$ to about 75 layers at lower temperature from which the peaks jump to the four layer repeat configuration known to correspond to the $\mathrm{SmC}_{\mathrm{F} 22}^{*}$ structure. They observed a continuous variation in the periodicity across the $\mathrm{SmC}_{\alpha}^{*}-\mathrm{SmC}^{*}$ transition. The layer spacing is observed to split above the $\mathrm{SmC}_{\alpha}^{*}-\mathrm{SmC}^{*}$ transition, an effect that was associated with a sample-degra-

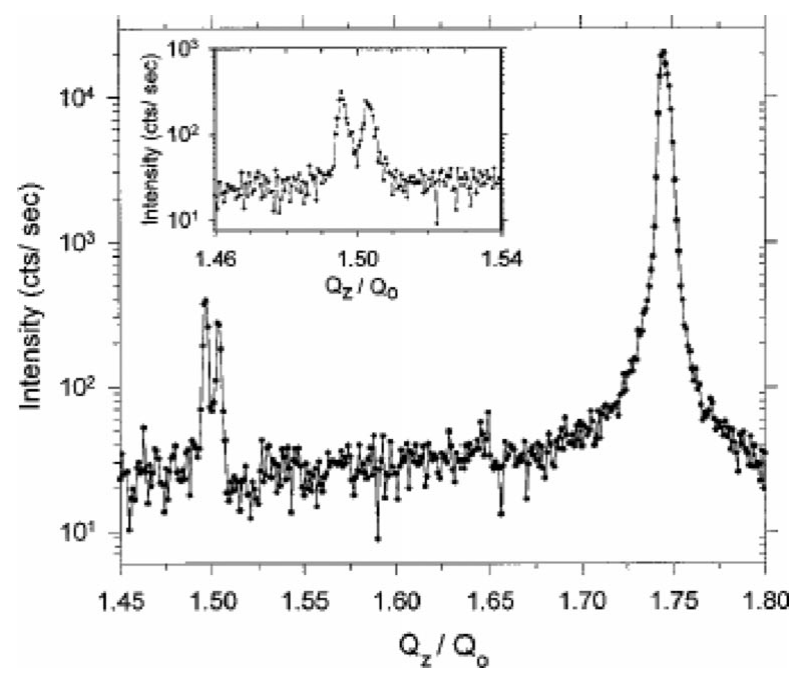

Figure 4

$\mathrm{X}$-ray intensity versus wavevector transfer for the $\mathrm{SmC}_{\mathrm{F} 12}^{*}$ phase of MHDDOPTCOB. The splitting becomes resolvable for the second-order peak. Reprinted figure with permission from Mach et al. (1999). Phys. Rev. E, 60, 6793-6802. Copyright (1999) by the American Physical Society. dation-induced $\mathrm{SmC}^{*}$ phase by Lagerwall et al. (2003) for a different antiferroelectric material. In the MHPOBC case, the layer spacing splitting occurred for all the tilted phases while for the new material, studied with resonant techniques, the layer-spacing splitting occurred mainly in the $\mathrm{SmC}_{\alpha}^{*}$ phase. Hirst et al. (2002) suggest the splitting is likely to be due to temperature gradients or surface effects.

The $\mathrm{SmC}_{\mathrm{Fl} 2}^{*}$ phase (Fig. 3) was observed in 10OTBBB1M7 (Mach et al., 1998) and in MHDDOPTCOB (Mach et al., 1999). Lorman (1996) showed that the $\mathrm{SmC}_{\mathrm{FI} 2}^{*}$ phase could involve a structure which is either uniaxial or biaxial about the layer normal. The former has a $90^{\circ}$ rotation from one layer to the next while the latter rotates alternately by $\delta$ and $180^{\circ}-\delta$. The early resonant X-ray results supported a uniaxial structure while ellipsometry measurements (Johnson et al., 2000) showed that the structure was biaxial. Cady et al. (2001) confirmed using higher-resolution resonant X-ray diffraction that it was indeed biaxial, with the additional modification of constant interlayer rotation on $\sim 1 \mu \mathrm{m}$ scale. The data and structure factors were in good agreement. The combination of two modulations results in the splitting of resonant reflections shown in Fig. 4.

The three plane repeat $\mathrm{SmC}_{\mathrm{FI} 1}^{*}$ phase has been studied for several materials (Mach et al., 1998, 1999; Hirst et al., 2002). The three plane order and predicted polarization have been confirmed (Mach et al., 1999). Fine structure was observed in the resonant peak but this did not correspond to the splitting due to the long helical pitch.

A wealth of information is now available to guide understanding of these phases. The Ising models for the SmC* phases, which suggest a single tilt plane with combinations of 0 and $\pi$ rotation angles, are ruled out by the X-ray results (Mach et al., 1998). Čepič \& Žekš (2001) have created a phenomenological model which is consistent with the observed $\mathrm{SmC}_{\mathrm{FI} 2}^{*}$ and $\mathrm{SmC}_{\mathrm{F} 1}^{*}$ structures. The key observation is that the existence of subphases is highly sensitive to the optical purity of the sample. This model includes a variety of interactions: steric, van der Waals, electrostatic, piezoelectric, flexoelectric between nearest layers and electrostatic between next-nearest layers. The cause and role of the discrete flexoelectric effect has been elaborated by Emelyanenko \& Osipov (2003). Using a similar model, they predict the observed phases and additionally subphases with longer plane sequences, which have not yet been observed.

Without establishing a specific model, Lagerwall et al. (2003) suggest that the chirality is not the key ingredient. Using a range of examples, they point out that antiferroelectric subphase suppression can be due to mixing induced steric and polar interactions with the role of chirality being less important. Related observations are made: (i) that the short plane stacking sequences and the $\mathrm{SmC}_{\mathrm{A}}^{*}$ phase are incompatible with the interdigitation of layers that is common in smectic materials; (ii) that $\mathrm{SmC}_{\mathrm{A}}^{*}$ order is incompatible with the existence of a high-temperature $\mathrm{N}^{*}$ phase; instead, I-SmA transitions occur. From this pair of observations, it is concluded that the tendency to form discrete layers is very strong in these materials. In addition, these phases are only 
observed for materials with large dipoles and occur when the tilt angle is large. The suggestion is that electrostatic and steric effects dominate with chirality playing a less important role. A full model for the range of observed behaviour is not yet available. That this approach could explain the $\mathrm{SmC}_{\mathrm{F} 22}^{*}$ and $\mathrm{SmC}_{\mathrm{FI} 1}^{*}$ structures is contested (Osipov, 2004).

\section{Smectic phases within gel structures}

In the studies reviewed in the previous two sections, complicated phase-transition behaviour was observed in singlecomponent systems. In the studies reviewed in this section, the novelty arises due to the two different components: the liquid crystal and the gel structure. The effect of disorder on the SmA state is fascinating because the state itself lacks true long-range order. Fluctuations in the layer position diverge logarithmically with the size of the system. This is known as the Landau-Peierls instability and was confirmed by AlsNielsen et al. (1980). The results of SmA liquid crystals in gels are compared with bulk liquid-crystal behaviour and with theoretical predictions. The bulk phenomena of importance are the coupling between the $\mathrm{N}$ and SmA phases and the anomalous elasticity exhibited by the SmA phase. The important theoretical predictions are those of the randomfield model (Imry \& Ma, 1975) and those due to Radzihovsky \& Toner (1999). The bulk liquid-crystal features and the theoretical models are introduced in the following paragraphs.

The N-SmA transition has unusual critical properties due to the coupling between $\mathrm{N}$ and $\mathrm{SmA}$ order parameters and fluctuations. If the $\mathrm{N}-\mathrm{SmA}$ transition occurs close in temperature to the $\mathrm{I}-\mathrm{N}$ transition, then the $\mathrm{N}$ order is strongly enhanced by the SmA order (Garland \& Nounesis, 1994). This coupling leads to a tricritical point (McMillan, 1971; de Gennes \& Prost, 1993). Conversely, if the N order is fully saturated before the $\mathrm{N}-\mathrm{SmA}$ transition, then the critical properties more closely resemble those for a two-component order parameter $(X Y)$ system in three dimensions (3D). Coupling between the $\mathrm{N}$ fluctuations and the SmA order parameter leads to an anisotropic critical regime. This picture has recently been confirmed with unprecedented precision via $\mathrm{X}$-ray studies of samples aligned in a $5 \mathrm{~T}$ magnetic field (Primak et al., 2002a,b). The theory of this behaviour is not yet complete. Any changes to these couplings due to the gel environment may well be instructive.

Anomalous elasticity in bulk SmA materials was first discussed by Grinstein \& Pelcovits (1981). Displacements in the layering direction and those perpendicular to the layers are coupled due to the non-linear stress-strain relation (de Gennes \& Prost, 1993). Owing to the Landau-Peierls instability, there are always angular fluctuations. Hence, when the stress is zero, the displacements in the in-plane direction cause displacements in the layering direction. This modifies the layer thickness. An imposed stress can easily be accommodated by suppressing the tilt fluctuations. A related effect occurs for the splay. The compression, $B$, and splay, $K_{1}$, elastic constants vanish and diverge, respectively, on long length scales. Disorder may modify the tilt fluctuations and this may be reflected in the elastic properties.

Two models for the effect of the gel are considered. The first is the random-field model (Larkin \& Ovchinnikov, 1979) corresponding to the pinning of the layer position by the random disorder. The $\mathrm{N}-\mathrm{SmA}$ transition is described by a twocomponent order parameter $\psi(\mathbf{r})=|\psi(\mathbf{r})| \exp \left[i q_{0} u(\mathbf{r})\right]$, where $|\psi(\mathbf{r})|$ is the strength of the density modulation, $q_{0}=2 \pi / d$, where $d$ is the layer periodicity, and $u(\mathbf{r})$ is the layer displacement away from perfect order. The contribution to the smectic free-energy density is

$$
f_{\mathrm{rf}}=-\operatorname{Re}\{V(\mathbf{r}) \psi(\mathbf{r})\} .
$$

The random disorder, $V(\mathbf{r})$, pins the phase of the density wave. These random fields would be expected to destroy the ordered state, replacing it with finite-sized domains (Imry \& Ma, 1975). The size of the domains and the structure factor are predicted to vary systematically with the variance of the layer pinning field (Aharony \& Pytte, 1983). The changes depend on the lower marginal dimensionality of the system.

The second model is due to Radzihovsky \& Toner (1999). They emphasize two important effects of the gel on the liquid crystal: the pinning of the position of the smectic layers and the pinning of the orientation of the nematic director. The contribution to the smectic free-energy density is

$$
f_{\text {gel }}=f_{\text {rf }}-[\mathbf{g}(\mathbf{r}) \cdot \hat{\mathbf{n}}(\mathbf{r})]^{2} .
$$

In this case, $\mathbf{g}(\mathbf{r})$ represents a random tilt effect imposed by the gel on the director. The effect of the field, $V(\mathbf{r})$, is to destroy the ordered phase, replacing it with finite-sized ordered domains since this is the random-field model described above. The effect of the field, $\mathbf{g}(\mathbf{r})$, which pins the orientation of the nematic director, is to perturb the smectic order via the interaction between the two order parameters and their fluctuations. Radzihovsky \& Toner (1999) found that the effect of disorder on the director would produce novel changes on the smectic state. A new smectic Bragg glass state was predicted characterized by short-range smectic order, glassy dynamics and enhanced anomalous elasticity. Fluctuations created by the perturbation of the director strongly enhance the anomalous elasticity effect (Radzihovsky \& Toner, 1999). This model predicts that the correlation length should have a temperature dependence related to that of the layer compression modulus, $B$ (Bellini et al., 2001).

In the studies reviewed here, liquid crystals are confined in chemically bonded porous silica structures (aerogels) or alternatively in a weakly bonded gel formed by hydrogen bonds between silica nanoparticles (aerosils). With increasing quantities of silica, the samples became progressively more opaque to X-rays. The availability of high-brilliance synchrotron X-ray sources has facilitated the study of liquid-crystal phase transitions within a silica gel environment.

\subsection{N-SmA transition in gels}

The N-SmA transition of $8 \mathrm{CB}$ was studied using X-ray diffraction within a rigid silica aerogel (Clark et al., 1993; 
Rappaport et al., 1996; Bellini et al., 2001). The N-SmA transition was observed to be destroyed as seen by the broadening of the corresponding X-ray reflection. Comparisons of the temperature dependence of the correlation length and peak amplitude suggested that the $8 \mathrm{CB}$-aerogel system is close to the novel smectic Bragg glass state (Bellini et al., 2001). It was subsequently a surprise to find that weakening the disorder by changing from aerogel to aerosil did not move the system closer to this novel soft state.

High-resolution X-ray diffraction studies were carried out on 8CB-aerosil gels (Park et al., 2002; Leheny et al., 2003) and 8OCB-aerosil gels (Clegg, Stock et al., 2003). The gel is fabricated from aerosils that are hydrophilic silica nanoparticles. These are dispersed in the liquid crystal and they hydrogen bond together to create a very low density gel structure. The gel percolates above a very low threshold volume fraction and the liquid-crystal gel forms a soft composite. A range of gel densities was explored over the temperature range of the $\mathrm{N}-\mathrm{SmA}$ transition. The smectic correlation length was found to saturate at a finite value at low temperatures and this value depended on the density of the gel structure (Fig. 5). Both the 3D- $X Y$ random-field model and the model due to Radzihovsky \& Toner (1999) predict that the structure factor characterizing the smectic fluctuations will be altered in the gel. The solid lines in Fig. 5 are the result of fits of the anticipated two-component line shape to $8 \mathrm{OCB}$-aerosil data. The finite correlation length indicates that the QLRO SmA phase has been destroyed, although there remains a pseudo-transition between a high-temperature regime where thermal fluctuations dominate and a low-temperature regime where static quenched-random effects dominate. A further study was carried out on the highly anisotropic liquid crystal

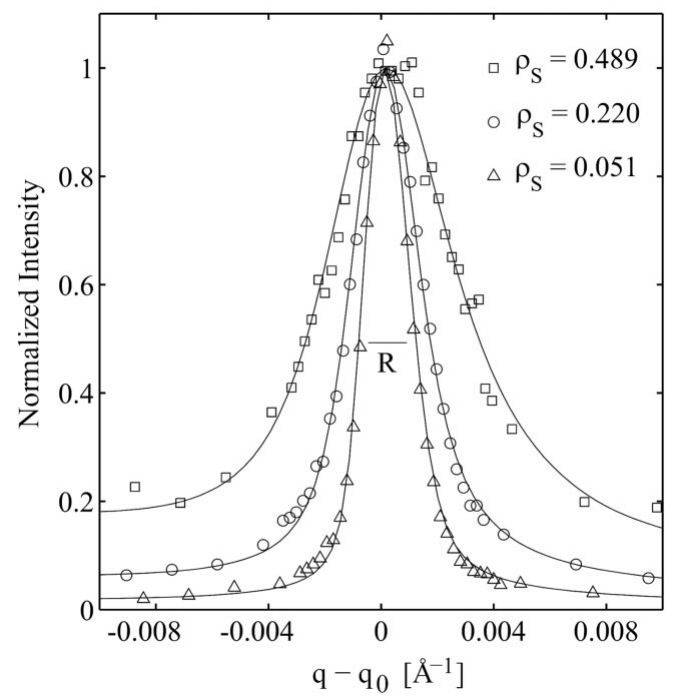

Figure 5

The broadening of the normalized X-ray reflection from SmA order due to the influence of the aerosil gel structure. In this case, the results are for $8 \mathrm{OCB}-$ aerosil at low temperatures (approximately $T_{\mathrm{NA}}^{0}-25 \mathrm{~K}$ ). The horizontal line labelled $R$ is the width of the instrumental resolution. The model of the effect of the gel is shown by the lines through the points. Reprinted figure with permission from Clegg et al. (2003). Phys. Rev. E, 67, 021703-1-13. Copyright (2003) by the American Physical Society. $\overline{8}$ S5 in an aerosil gel (Clegg, Birgeneau et al., 2003). The twocomponent line shape also gave a very good account of these results. A low-temperature correlation length could not be extracted in this case due to the intervention of the SmA-SmC phase transition.

The correlation lengths at low temperature for $8 \mathrm{CB}$-aerosil and $8 \mathrm{OCB}$-aerosil are plotted in Fig. 6 as a function of the density of the gel ( $\rho_{S}=$ mass of silica/volume of liquid crystal). Since each new particle perturbs the average phase which is favourable to the existing aerosils, the gel density is taken to be a measurement of the variance of the random field. The solid lines in Fig. 6 are the expected behaviour according to the 3D- $X Y$ random-field model (Aharony \& Pytte, 1983). The good agreement suggests that layer pinning may be the dominant effect in these systems.

Comparisons with the random-field model have been augmented by more detailed observations. Systematic changes in the pseudo-critical properties have been recorded in all cases. This is most evident using ac-calorimetry (Zhou et al., 1997; Iannacchione et al., 1998; Clegg, Stock et al., 2003). The heat capacity exponent, $\alpha$, reflects the $\mathrm{N}$ susceptibility at the $\mathrm{N}-\mathrm{Sm} A$ transition. High $\mathrm{N}$ susceptibility gives an $\alpha$ close to the tricritical value. Low $\mathrm{N}$ susceptibility implies little coupling between $\mathrm{N}$ and SmA order parameters and $\alpha$ approaches the $3 \mathrm{D}-X Y$ value. For the N-SmA transition in an aerosil gel, $\alpha$ moves toward the $3 \mathrm{D}-X Y$ value with increasing gel density. Comparable behaviour is observed for the disconnected susceptibility as studied using X-ray diffraction (Leheny et al., 2003; Iannacchione et al., 2003). The evidence suggests that the $\mathrm{N}-\mathrm{SmA}$ anisotropic criticality moves toward $3 \mathrm{D}-X Y$ pseudocriticality in the presence of quenched random disorder. This observation is consistent with the gel causing the $\mathrm{N}$ suscep-

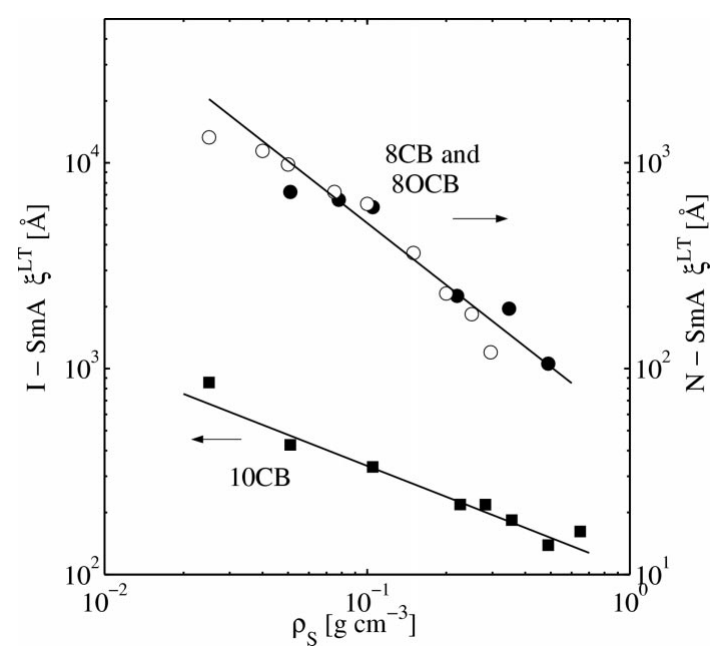

Figure 6

Cube root of the SmA correlation volume, $\bar{\xi}$, taken at low temperature versus the gel density, $\rho_{S}$, for $\mathrm{N}-\mathrm{SmA}$ and I-SmA transitions. The solid lines represent single power laws that are different for the two types of transition. The open circles are for 8CB-aerosil (Leheny et al., 2003), the solid circles are for 8OCB-aerosil (Clegg, Stock et al., 2003) and the solid squares are for 10CB--aerosil (Ramazanoglu et al., 2004). Reprinted figure with permission from Ramazanoglu et al. (2004). Phys. Rev. E, 69, 061706-1-8. Copyright (2004) by the American Physical Society. 
tibility to decrease toward zero. This effect is not currently part of either theoretical model.

One of the key differences between X-ray results for the $\mathrm{N}-\mathrm{SmA}$ in aerogel and aerosil is the temperature dependence of the correlation lengths and the susceptibility. Both show these quantities saturating at finite values at low temperature. In aerogel, the temperature dependence in the transition region is strongly altered from bulk behaviour and this has been interpreted to suggest enhanced anomalous elasticity (Bellini et al., 2001). By contrast, with aerosil the temperature dependence in the transition region is only subtly modified from bulk behaviour (Leheny et al., 2003; Iannacchione et al., 2003). The modification correlates well with ac-calorimetry results.

\subsection{The role of nematic order}

The apparent schism between aerogel and aerosil results motivated further studies particularly concerning the role of nematic order. Ramazanoglu et al. (2004) studied the I-SmA transition in 10CB-aerosil where there is no orientational order prior to the formation of SmA order. By contrast, Liang et al. (2004) studied the N-SmA transition in an anisotropic aerosil gel. In this case, the liquid crystal has long-range orientational order prior to the onset of SmA order. These studies will be described in turn.

In order to probe the interaction between the orientational order and the gel environment, Ramazanoglu et al. (2004) studied 10CB-aerosil gels. Bulk $10 \mathrm{CB}$ has a strongly firstorder transition directly from an I phase into the SmA phase. In the aerosil gel, the SmA correlation length, extracted using the two-component line-shape model, was found to saturate at low temperatures and to decrease systematically with increasing gel density. As shown in Fig. 6, the density dependence was $1 / \sqrt{ } \rho_{S}$ for the I-SmA transition while it was $1 / \rho_{S}$ for the N-SmA transition. This difference, resulting from the presence of nematic order, is consistent with the random-field model. The change is associated with the fact that the nematic director is forced to point in the direction of the layer normal (Ramazanoglu et al., 2004). Preliminary measurements were made on the I-SmA transitions for $10 \mathrm{CB}$ and $650 \mathrm{BC}$ in rigid aerogel (Bellini et al., 1996, 2003). The correlation lengths are observed to increase discontinuously at the transition temperature and to saturate quickly. The scattering intensity increases only slowly with decreasing temperature. The results appear very similar to those for aerosil gels at high $\rho_{s}$. Only a single aerogel pore size was studied, making a full comparison with the aerosil results difficult at present.

Liang et al. (2004) created anisotropic gels by training standard liquid-crystal aerosil gels in a magnetic field. The idea for this method followed from the deuterium NMR studies by Jin \& Finotello (2001). The alignment of the molecules by the field led to the gradual but permanent rearrangement of the gel structure. This technique resulted in anisotropic gels provided the aerosil density $\rho_{S} \leq 0.1 \mathrm{~g} \mathrm{~cm}^{-3}$. High-resolution X-ray studies were carried out on $8 \mathrm{CB}$ in an anisotropic gel across the temperature range of the $\mathrm{N}-\mathrm{SmA}$ transition. In part, this research was to test a theoretical prediction that an $X Y$ Bragg glass phase will be stabilized in smectics with anisotropic disorder (Jacobsen et al., 1999). This prediction is for the case of disorder which has been stretched so that the nematic director has a preferred orientation. This situation suppresses long length-scale nematic fluctuations. The low-temperature state should then be an $X Y$ Bragg glass state with algebraically decaying correlations.

The studies showed that long-range nematic order was created by training the gels as seen via the anisotropy in Fig. 7 . The SmA correlations that developed below the pseudotransition were not characteristic of the anticipated $X Y$ Bragg glass state (Liang et al., 2004). The behaviour through and below the pseudo-transition has many similarities with the behaviour of $8 \mathrm{CB}$-aerosil when the gel is isotropic. In the trained case, the signal-to-noise ratio is highly favourable since the sample is a single crystal. The structure factor was found to be modified slightly compared with the model for the untrained system. The anisotropic scaling of correlation lengths is suppressed when the random fields are imposed. This is indicative of the anisotropic N-SmA fixed point becoming more like $3 \mathrm{D}-X Y$ behaviour in the presence of quenched disorder. It appears that both couplings between $\mathrm{N}$ and $\mathrm{SmA}$ order parameters and fluctuations are suppressed by the random environment.

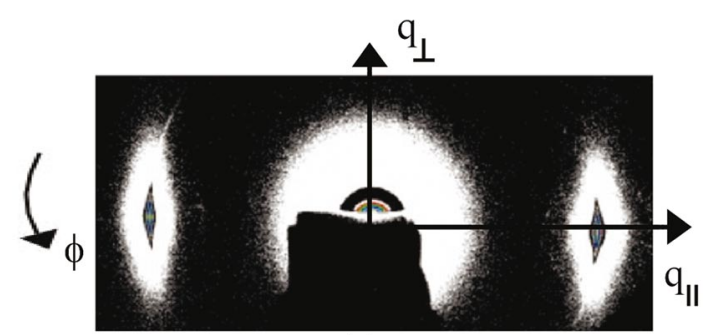

(a)

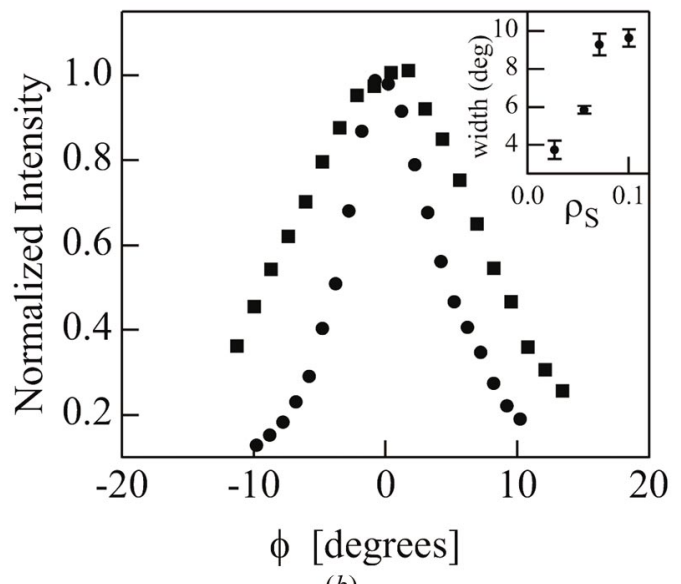

(b)

Figure 7

(a) X-ray intensity distribution from SmA correlations in an anisotropic aerosil gel $\left(\rho_{S}=0.035 \mathrm{~g} \mathrm{~cm}^{-3}\right)$. The underlying nematic order is long range while the SmA order is short range. $(b)$ The sample mosaic spread for anisotropic gel samples of density $\rho_{S}=0.027 \mathrm{~g} \mathrm{~cm}^{-3}$ (circles) and $\rho_{S}=0.100 \mathrm{~g} \mathrm{~cm}^{-3}$ (squares) at $300.1 \mathrm{~K}$. Reprinted figure with permission from Liang et al., 2004). J. Phys. Condens. Matter, 16, S1989-S2002. Copyright (2004) by the Institute of Physics Publishing. 
The series of measurements on the N-SmA transition in aerosil gels have failed to corroborate earlier indications of smectic Bragg glass behaviour in an aerogel. In addition, both $\mathrm{N}-\mathrm{SmA}$ and I-SmA transitions are modified in a manner consistent with a simple random-field model. It may be possible that the earlier conclusions with respect to the smectic Bragg glass (Bellini et al., 2001) were premature.

\section{Conclusions}

This review has shown the huge range of behaviour exhibited by smectic liquid crystals when they are perturbed by twist, dipole moments and local deformations. This has included a rich variety of phases and novel pseudotransition behaviour. Many of the observations are still incompletely understood.

Further developments using X-ray techniques are anticipated in the three areas described. In the field of twist-grainboundary studies, recent observations reveal further new phases, these have not yet been subjected to X-ray study in oriented samples and have been reviewed by Goodby (2002). The range includes $\mathrm{TGBC}_{\mathrm{A}}^{*}$ structures where the $\mathrm{SmC}_{\mathrm{A}}^{*}$ order is antiferroelectric (Goodby et al., 2000) and also re-entrant TGBA phases (Rao et al., 2001).

Resonant X-ray diffraction can now be applied to a much broader range of materials. One key advance was the demonstration that non-resonant mesogens could be studied using resonant $\mathrm{X}$-ray diffraction by mixing them with a small fraction of resonant liquid crystal (Hirst et al., 2002). The second advance was the application of resonant diffraction to the study of liquid crystals containing Se (Matkin et al., 2001). The energy of the $K$ edge $(12.66 \mathrm{keV})$ is sufficient to allow samples to be studied in the context of realistic electro-optic devices.

The stability of the smectic Bragg glass could be further probed by combining the $8 \mathrm{CB}$-aerogel samples studied by Bellini et al. (2001) with a high magnetic field. The effects initially observed should be enhanced as the $\mathrm{N}$ order becomes long range. Orienting the sample would also improve the signal-to-noise ratio without increasing the sample damage. Recent pioneering research includes new studies on $\mathrm{SmC}^{*}$ phases in an aerosil gel (Kutnjak et al., 2003) which have yet to be examined with X-ray diffraction.

I am grateful to S. Egelhaaf, C. Garland, H. Gleeson, G. Iannacchione, R. Leheny, M. Osipov and R. Sastri for helpful comments and to P. Barois, H. Gleeson, R. Leheny, L. Navailles and R. Pindak for providing figures. Funding in Edinburgh came from the EPSRC (Grant GR/S10377/01). Much of the research presented in $\$ 4$ was carried out at the University of Toronto and was funded by the Natural Sciences and Engineering Research Council of Canada.

\section{References}

Abrikosov, A. A. (1957). Sov. Phys. JETP, 5, 1174-1183. Aharony, A. \& Pytte, E. (1983). Phys. Rev. B, 27, 5872-5874.
Als-Nielsen, J., Litster, J. D., Birgeneau, R. J., Kaplan, M., Safinya, C. R., Lindegaard-Andersen, A. \& Mathiesen, S. (1980). Phys. Rev. $B, \mathbf{2 2}, 312-320$.

Als-Nielsen, J. \& McMorrow, D. (2001). Elements of Modern X-ray Physics. Chichester: John Wiley.

Bellini, T., Clark, N. A. \& Link, D. R. (2003). J. Phys. Condens. Matter, 15, S175-S182.

Bellini, T., Radzihovsky, L., Toner, J. \& Clark, N. A. (2001). Nature (London), 294, 1074-1079.

Bellini, T., Rappaport, A. G., Clark, N. A. \& Thomas, B. N. (1996). Phys. Rev. Lett. 77, 2507-2510.

Brunet, M., Navailles, L. \& Clark, N. A. (2002). Eur. Phys. J. E7, 5-11.

Cady, A., Pitney, J. A., Pindak, R., Matkin, L. S., Watson, S. J., Gleeson, H. F., Cluzeau, P., Barois, P., Levelut, A.-M., Caliebe, W., Goodby, J. W., Hird, M. \& Huang, C. C. (2001). Phys. Rev. E, 64, 050702(R)-1-4.

Čepič, M. \& Žekš, B. (1995). Mol. Cryst. Liq. Cryst. A263, 61-67.

Čepič, M. \& Žekš, B. (2001). Phys. Rev. Lett. 87, 085501-1-4.

Chan, T. \& Garland, C. W. (1995). Phys. Rev. E, 52, 5000-5003.

Clark, N. A., Bellini, T., Malzbender, R. M., Thomas, B. N., Rappaport, A. G., Muzny, C. D., Schaefer, D. W. \& Hrubesh, L. (1993). Phys. Rev. Lett. 71, 3505-3508.

Clegg, P. S., Birgeneau, R. J., Park, S., Garland, C. W., Iannacchione, G. S., Leheny, R. L. \& Neubert, M. E. (2003). Phys. Rev. E, 68, 031706-1-7.

Clegg, P. S., Stock, C., Birgeneau, R. J., Garland, C. W., Roshi, A. \& Iannacchione, G. S. (2003). Phys. Rev. E, 67, 021703-1-13.

Davidov, D., Safinya, C. R., Kaplan, M., Dana, S. S., Schaetzing, R., Birgeneau, R. J. \& Litster, J. D. (1979). Phys. Rev. B, 19, 1657-1663.

Dmitrienko, V. E. (1983). Acta Cryst. A39, 29-35.

Dozov, I. (1995). Phys. Rev. Lett. 74, 4245-4248.

Emelyanenko, A. V. \& Osipov, M. A. (2003). Phys. Rev. E, 68, 051703-1-15.

Fera, A., Opitz, R., de Jeu, W. H., Ostrovskii, B. I., Schlauf, D. \& Bahr, Ch. (2001). Phys. Rev. E, 64, 021702-1-8.

Fukuda, A., Takanishi, Y., Isozaki, T., Ishikawa, K. \& Takezoe, H. (1994). J. Mater. Chem. 4, 997-1016.

Furukawa, K., Tereshami, K., Ichihashi, M., Saitoh, S., Miyazawa, K. \& Inukai, T. (1988). Ferroelectrics, 85, 451-451.

Galerne, Y. (2000). Eur. Phys. J. E3, 355-368.

Garland, C. W. \& Nounesis, G. (1994). Phys. Rev. E, 49, 2964-2971.

Gennes, P. G. de (1972). Solid State Commun. 10, 753-756.

Gennes, P. G. de \& Prost, J. (1993). The Physics of Liquid Crystals, 2nd ed. New York: Oxford University Press.

Goodby, J. W. (2002). Curr. Opin. Colloid Inter. 7, 326-332.

Goodby, J. W., Petrenko, A., Hird, M., Lewis, R. A., Meier, J. \& Jones, J. C. (2000). J. Chem. Soc. Chem. Commun. pp. 1149-1150.

Goodby, J. W., Waugh, M. A., Stein, S. M., Chin, E., Pindak, R. \& Patel, J. S. (1989a). Nature (London), 337, 449-452.

Goodby, J. W., Waugh, M. A., Stein, S. M., Chin, E., Pindak, R. \& Patel, J. S. (1989b). Am. Chem. Soc. 111, 8119-8125.

Grinstein, G. \& Pelcovits, R. A. (1981). Phys. Rev. Lett. 47, 856-859.

Hiji, N., Chandani, A. D. L., Nishiyama, S., Ouchi, Y., Takezoe, H. \& Fukuda, A. (1988). Ferroelectrics, 85, 99-99.

Hirst, L. S., Watson, S. J., Gleeson, H. F., Cluzeau, P., Barois, P., Pindak, R., Pitney, J., Cady, A., Johnson, P. M., Huang, C. C., Levelut, A.-M., Srajer, G., Pollmann, J., Caliebe, W., Seed, A., Herbert, M. R., Goodby, J. W. \& Hird, M. (2002). Phys. Rev. E, 65, 041705-1-10.

Iannacchione, G. S., Garland, C. W., Mang, J. T. \& Rieker, T. P. (1998). Phys. Rev. E, 58, 5966-5981.

Iannacchione, G. S., Park, S., Garland, C. W., Birgeneau, R. J. \& Leheny, R. (2003). Phys. Rev. E, 67 011709-1-13.

Imry, Y. \& Ma, S.-K. (1975). Phys. Rev. Lett. 35, 1399-1401.

Jacobsen, B., Saunders, K., Radzihovsky, L. \& Toner, J. (1999). Phys. Rev. Lett. 83, 1363-1366.

Jin, T. \& Finotello, D. (2001). Phys. Rev. Lett. 86, 818-821. 
Johnson, P. M., Olson, D. A., Pankratz, S., Nguyen, T., Goodby, J., Hird, M. \& Huang, C. C. (2000). Phys. Rev. Lett. 84, 4870-4873.

Kamien, R. D. \& Lubensky, T. C. (1993). J. Phys. (Paris) I, 3, 2131-2138.

Kumar, S. (2001). Liquid Crystals, Experimental Study of Physical Properties and Phase Transitions, edited by S. Kumar. Cambridge University Press.

Kutnjak, Z., Cordoyiannis, G. \& Nounesis, G. (2003). Ferroelectrics, 294, 105-111.

Lagerwall, J. P. F., Rudquist, P., Lagerwall, S. T. \& Giesselmann, F. (2003). Liq. Cryst. 30, 399-414.

Larkin, A. I. \& Ovchinnikov, Yu. N. (1979). J. Low Temp. Phys. 34, 409-428.

Leheny, R. L., Park, S., Birgeneau, R. J., Gallani, J.-L., Garland, C. W. \& Iannacchione, G. S. (2003). Phys. Rev. E, 67, 011708-1-13.

Levelut, A.-M. \& Pansu, B. (1999). Phys. Rev. E, 60, 6803-6815.

Liang, D., Borthwick, M. A. \& Leheny, R. L. (2004). J. Phys. Condens. Matter, 16, S1989-S2002.

Lorman, V. L. (1995). Mol. Cryst. Liq. Cryst. A262, 437-453.

Lorman, V. L. (1996). Liq. Cryst. 20, 267-276.

Mach, P., Pindak, R., Levelut, A.-M., Barois, P., Nguyen, H. T., Baltes, H., Hird, M., Toyne, K., Seed, A., Goodby, J. W., Huang, C. C. \& Furenlid, L. (1999). Phys. Rev. E, 60, 6793-6802.

Mach, P., Pindak, R., Levelut, A.-M., Barois, P., Nguyen, H. T., Huang, C. C. \& Furenlid, L. (1998). Phys. Rev. Lett. 81, 1015-1018.

McMillan, W. L. (1971). Phys. Rev. A, 4, 1238-1246.

Matkin, L. S., Watson, S. J., Gleeson, H. F., Pindak, R., Pitney, J., Johnson, P. M., Huang, C. C., Barois, P., Levelut, A.-M., Srajer, G., Pollmann, J., Goodby, J. W. \& Hird, M. (2001). Phys. Rev. E, 64, 021705-1-6.

Meyer, R. B., Liebert, L., Strzelecki, L. \& Keller, P. (1975). J. Phys. (Paris) Lett. 36, L69-L71.

Navailles, L., Barois, P. \& Nguyen, H. T. (1993). Phys. Rev. Lett. 71, 545-548.

Navailles, L., Pansu, B., Gorre-Talini, L. \& Nguyen, H. T. (1998). Phys. Rev. Lett. 81, 4168-4171.

Navailles, L., Pindak, R., Barois, P. \& Nguyen, H. T. (1995). Phys. Rev. Lett. 74, 5224-5227.

Nguyen, H. T., Bouchta, A., Navailles, L., Barois, P., Isaert, N., Tweig, R. J., Maaroufi, A. \& Destrade, C. (1992). J. Phys. (Paris) II, 2, 1889-1906.

Osipov, M. A. (2004). Private communication.

Park, S., Leheny, R. L., Birgeneau, R. J., Gallani, J.-L., Garland, C. W. \& Iannacchione, G. S. (2002). Phys. Rev. E, 65, 050703(R)-1-4.
Pershan, P. S. (1988). Structure of Liquid Crystal Phases. Singapore: World Scientific.

Petit, M., Barois, P. \& Nguyen, H. T. (1996). Europhys. Lett. 36, 185-190.

Pikin, S. A., Hiller, S. \& Haase, W. (1995). Mol. Cryst. Liq. Cryst. A262, 425-435.

Pramod, P. A., Pratibha, R. \& Madhusudana, N. V. (1997). Curr. Sci. 73, 761-765.

Primak, A., Fisch, M. \& Kumar, S. (2002a). Phys. Rev. Lett. 88, 035701-1-4.

Primak, A., Fisch, M. \& Kumar, S. (2002b). Phys. Rev. E, 66, 0517071-1-13.

Radzihovsky, L. \& Toner, J. (1999). Phys. Rev. B, 60, 206-257.

Ramazanoglu, M. K., Clegg, P. S., Birgeneau, R. J., Garland, C. W., Neubert, M. E. \& Kim, J. M. (2004). Phys. Rev. E, 69, 061706-1-8.

Rao, D. S. S., Prasad, S. K., Raja, V. N., Yelemaggad, C. V. \& Nagamani, S. A. (2001). Phys. Rev. Lett. 87, 085504-1-4.

Rappaport, A. G., Clark, N. A., Thomas, B. N. \& Bellini, T. (1996). Liquid Crystals in Complex Geometries Formed by Polymer and Porous Networks, edited by G. P. Crawford \& S. Zumer. London: Taylor and Francis.

Renn, S. R. (1992). Phys. Rev. A, 45, 953-973.

Renn, S. R. \& Lubensky, T. C. (1988). Phys. Rev. A, 38, 2132-2147.

Renn, S. R. \& Lubensky, T. C. (1991). Mol. Cryst. Liq. Cryst. 209, 349-355.

Ribeiro, A. C., Barois, Ph., Galerne, Y., Oswold, L. \& Guillon, D. (1999). Eur. Phys. J. B11, 121-126.

Roy, A. \& Madushudana, N. (1996). Europhys. Lett. 36, 221-226.

Safinya, C. R., Kaplan, M., Als-Nielsen, J., Birgeneau, R. J., Davidov, D., Litster, J. D., Johnson, D. L. \& Neubert, M. E. (1980). Phys. Rev. $B, 21,4149-4153$.

Seddon, J. M. (1998). Handbook of Liquid Crystals, Vol. 1, edited by D. Demus, J. Goodby, G. W. Gray, H.-W. Spiess \& V. Vill. Weinheim: Wiley-VCH.

Shashidhar, R., Ratna, B. R., Nair, G. G., Prasad, S. K., Bahr, Ch. \& Heppke, G. (1988). Phys. Rev. Lett. 61, 547-549.

Takanishi, Y., Ikeda, A., Takezoe, H. \& Fukuda, A. (1995). Phys. Rev. E, 51 400-406.

Templeton, D. H. \& Templeton, L. K (1982). Acta Cryst. A38, 62-67.

Ybert, C., Navailles, L., Pansu, B., Rieutord, F., Nguyen, H. T. \& Barois, P. (2003). Europhys. Lett. 63, 840-845.

Zhou, B., Iannacchione, G. S. \& Garland, C. W. (1997). Phys. Rev. E, 55, 2962-2968. 